\title{
An Agent-based Negotiation Model to Support Partner Selection in a Virtual Enterprise
}

\author{
C.X. Yu, T.N. Wong, G. Wang \\ Department of Industrial and Manufacturing Systems Engineering \\ The University of Hong Kong \\ Hong Kong, China \\ cxyu@hkusua.hku.hk, tnwong@hku.hk,wanggong@hkusua.hku.hk
}

\begin{abstract}
-in response to the global business competitive environment, it is common for several companies to participate in a virtual enterprise (VE) to cooperate and collaborate dynamically to complete a common business opportunity. This paper proposes an agent-based negotiation model to support the partner selection process in a VE. To begin with, the VE partner selection problem is abstracted as a buyer-seller relationship such that the VE initiator is the buyer and the VE partners are sellers or vice verse. In the multi-agent system (MAS) that supports the proposed negotiation model, autonomous agents are established to represent various parties and functions of the VE. For instance, a buyer agent represents the $V E$ initiator and the potential partners are represented by seller agents. Thus, the VE partner evaluation and selection problem is the process of finding the partners that are able to provide the buyer with the right quality products and services at the right price and at the right time. Evaluation and selection of partners is a typical multiattribute decision making (MADM) problem involving various issues that can both be qualitative and quantitative.
\end{abstract}

Keywords- Multi-agent system, negotiation, partner selection, virtual enterprise, multiple inter-dependences

\section{INTRODUCTION}

With the fierce competition in today's global market, it is difficult for enterprises to fulfill business opportunities by themselves due to the constraints of technologies and resources. It becomes popular for several companies to work together as a virtual enterprise (VE), to accomplish business opportunity cooperatively. A VE can be defined as a temporary alliance of independent and geographically dispersed enterprises set up to share skills or core competencies and resources, in order to respond to business opportunities, the cooperation among the enterprises being supported by computer networks [1].

The enterprise that initiates a VE is the VE initiator, and enterprises that VE initiator cooperates with to accomplish specific short term goals are VE partners. Thus, partner selection of a VE is a process that VE initiator selects partners providing right quality products and services at the right price and at the right time. Each member enterprise will provide its own core competences in areas such marketing, engineering, and manufacturing to the VE. The performances of partners determine the competitive ability of a VE. So partner selection is an important problem of VE. Therefore, effective approaches for selecting partners in a VE are essential.

In partner selection of a VE, many criteria should be taken into consideration. Dickson[2], Weber et al.[3] and Zhang et al.[4] reviewed, classified and summarized articles on vendor selection, supplier selection and partner selection, and illustrated multiple criteria and effective methods for selection. In general, all these researchers pointed out that the partner selection problem involves multiple criteria including, attractive price, high quality, in time delivery, perfect post-sale service and so on. The partner selection problem has attracted many researchers' attention. Some researchers attempted the VE partner selection problem with optimization algorithms [58]. They pointed out that price is not the only criterion in partner selection problem, and they all viewed partner selection problem as a multi-attribute decision making (MADM) problem that involves trade-offs between conflicting criteria. Although researchers have presented different mathematical approaches to evaluate and select partners, they all assume that the multiple criteria are independent and once partners propose their proposals they can not change them any more.

However, in reality, inter-dependences between criteria exist. In addition, potential partners want to communicate with VE initiator and adjust their proposals according to situation in partner selection process. The main objective of this paper is to propose a new partner selection model that considers interdependences between criteria and supports communication between VE initiator and potential partners. In the proposed model, VE partner selection is achieved through agent-based negotiations in a multi-agent system (MAS). MAS is an agentbased system that comprises several interacting agents. According to the distributed, cooperative and intelligent characteristics of MAS, it is suitable to model a VE as a distributed MAS[9]. In the MAS, autonomous agents are established to represent various parties and functions of the VE. Agents interact to negotiate on the supply and demand of product or service.

The remainder of this paper is organized as follows. Section 2 describes the workflow and framework of proposed negotiation-based MAS that supports partner selection in a VE. In section 3, negotiation issue, utility function, and negotiation protocol and strategies of proposed negotiation model are presented. A simulation of the proposed negotiation model and 
experimental results are described in Section 4. Finally, conclusions and future research plans follow in section 5 .

\section{WORKFLOW AND FRAMEWORK OF NEGOTIATION-BASED MULTI-AGENT SYSTEM}

\section{A. Problem description}

With increasing global competition, it is common for manufacturing enterprises to distribute non-core business portions to collaborative companies. For example, automobile manufacturers usually outsource auto parts globally instead of producing by themselves. In this scenario, an automobile manufacturer and its cooperative auto parts manufacturers constitute a VE to complete vehicle production. In this VE, the automobile manufacturer is the initiator, and the cooperative auto parts manufacturers are partners. Typically, a large number of auto parts are involved in vehicle production. For each auto part, a lot of manufacturers are able to supply the product. It is necessary for the automobile manufacturer to select the combination of manufacturers for all auto parts. So the partner selection problem is rather complex. As an example, an automobile manufacturer has to procure 5 engine parts such as crank shafts and bearings. There are 20 suppliers which are able to supply all the 5 parts. To identify the combination of suppliers for the 5 engine parts, the partner selection solution number will involve 205 possible combinations. Therefore, it is appropriate for the automobile manufacturer to screen out several eligible companies, and then evaluates and negotiates with these eligible companies to form a VE.

Through this approach, an automobile manufacturer is only required to negotiate with a small number of potential partners to obtain the best possible proposal. An optimization procedure is suitable to select several best partner selection solutions and develop a likely partners shortlist for future negotiation. In the optimization procedure, the relationships between different auto parts manufacturers can be considered. For example, companies have good cooperation history are preferred. In this paper, particle swarm optimization (PSO) algorithm is used in experiments to screen out potential partners for negotiation. PSO bases on the ideas of animal flocking behavior, and it is primarily suited for numerical optimization problems. At first, the algorithm is simple, fast and very easy to code. Secondly, it is not computationally intensive in terms of memory requirements and time. Furthermore, it has fewer parameters to tune when compare with other optimization algorithms such as GA. So PSO algorithm is used to find best partner selection solutions and develop likely partners shortlist for each component.

Subsequently, agents have to engage in negotiation to discuss with potential partners on the shortlist and try to obtain a satisfying proposal. It is common to find that the potential partner in the last place of the shortlist is selected as partners. If the number of potential partners for an auto part is larger than the given length of shortlist, the optimization procedure is triggered to select likely partners to form the shortlist. Otherwise the automobile manufacturer negotiates with the potential partners directly to reach agreement. In this paper, the length of shortlist is three. The workflow of the negotiationbased MAS is shown in Fig. 1.

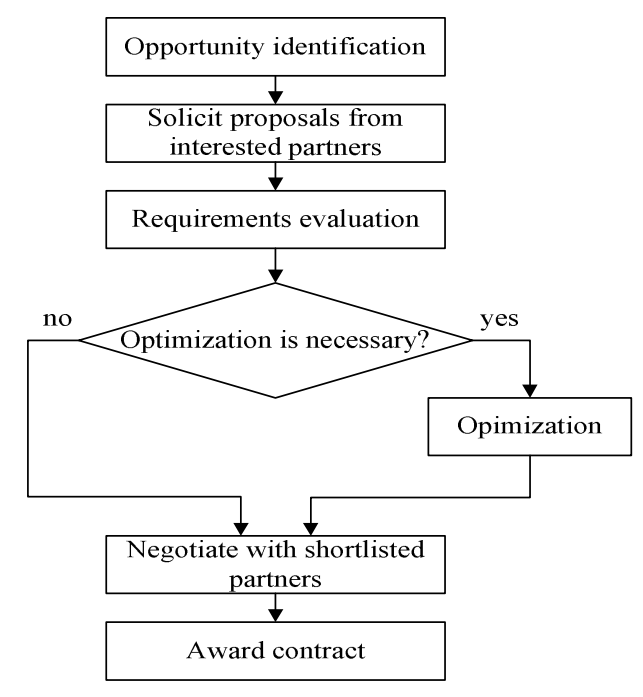

Figure 1. Workflow of negotiation-based MAS

\section{B. Framework of negotiation-based MAS}

The partner selection problem in a VE can be abstracted as a buyer-seller relationship such that the VE initiator is the buyer and the VE partners are sellers or vice verse. The VE is modeled as a distributed MAS. In the MAS, the buyer agent represents the $\mathrm{VE}$ initiator and the potential partners are represented by the seller agents. In the scenario that an automobile manufacturer selects suitable auto parts manufacturers, the automobile manufacturer is the buyer agent and auto parts manufacturers are seller agents. In the proposed negotiation model, partner selection for the automobile manufacturer is represented by one-to-many negotiation on multiple inter-dependent issues and the one-to-many negotiation is realized by several one-to-one negotiations.

Fig. 2 shows the framework of the proposed negotiationbased MAS from buyer's point of view. The process of partner selection is supported by eight types of agents, namely, Buyer Interface Agent (BIA), Buyer Agent (BA), Coordinator Agent (CA), Requirements Evaluation Agent (REA), Optimization Agent (OA), Knowledge Management Agent (KMA), Seller Agent (SA) and Seller Interface Agent (SIA).

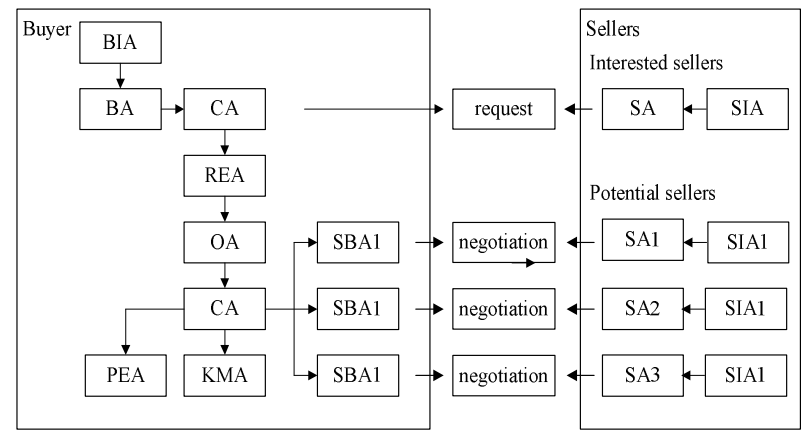

Figure 2. Framework of negotiation-based MAS 
- $\quad$ BIA and SIA are the interfaces between buyer or seller agent and users. Users set their requirements and preferences through these interfaces.

- $\mathrm{CA}$ is responsible for coordinating the activities of REA, OA, KMA, BA and SA. It can receive VE initiator's requirements, and query Buyer's knowledge to support the negotiation between BA and SA. In addition, CA also can control the concurrent one-toone negotiations between BA and SA.

- REA is used to filter SAs that do not satisfy the requirements of $\mathrm{BA}$.

- $\quad$ OA is used when the number of qualified SAs is large. It can select a given number of qualified SAs for future negotiation.

- KMA is designed for reserving the knowledge of buyer, for example, negotiation protocol, negotiation strategies, bid evaluation function and counter-offer generation methods.

- Performance Evaluation Agent (PEA) is responsible for evaluating the performance of each seller after cooperation.

- SubBuyer Agent (SBA) is the instance of BA created by $\mathrm{CA}$ to negotiate with each $\mathrm{SA}$. BA is responsible for reserving the information of buyer, controlling the action of buyer, negotiating with $\mathrm{SA}$, and reaching agreements. SA is the counterpart of BA, and has similar functionalities.

\section{AGENT-BASED NEGOTIATION MODEL FOR PARTNER SELECTION}

The proposed negotiation model is a one-to-many negotiation on multiple inter-dependent issues. The multiple issues can be expressed in qualitative and quantitative forms. In the negotiation process, both buyer agent and seller agent do not know each other's information such as utility function. Therefore, the negotiation setting can be described as doublesided incomplete information. Generally, there are three broad areas needed to be considered when building a negotiation model: negotiation issues, negotiation protocol and negotiation strategies.

\section{A. Negotiation issues}

In partner selection of a VE, negotiation issues are the criteria that evaluate potential partners. According to the actual demand of automobile manufacturers, the price issue, the quality issue, the delivery issue and the service issue are considered in the proposed negotiation model. In order to facilitate the expression of the decision-makers' preferences or assessments of potential partners efficiently under a multi-issue perspective, the four issues can be expressed in different forms. For example, quality issue and service issue are expressed in qualitative form, while price issue and delivery issue are expressed in quantitative form. For qualitative issues quality and service, five linguistic states are selected and expressed by appropriate fuzzy sets which are \{very poor, poor, medium, good, very good $\}$. The definition of triangular fuzzy numbers
(TFN) can be seen in table I and the membership function is shown in Fig. 3.

TABLE I. TFNS OF QUALITY AND SERVICE

\begin{tabular}{|c|c|}
\hline Linguistic variable & TFNs \\
\hline Very poor $(\mathrm{VP})$ & $(0,10,20)$ \\
Poor(P) & $(10,30,50)$ \\
Medium $(\mathrm{M})$ & $(30,50,70)$ \\
Good $(\mathrm{G})$ & $(50,70,90)$ \\
Very good $(\mathrm{VG})$ & $(80,90,100)$ \\
\hline
\end{tabular}

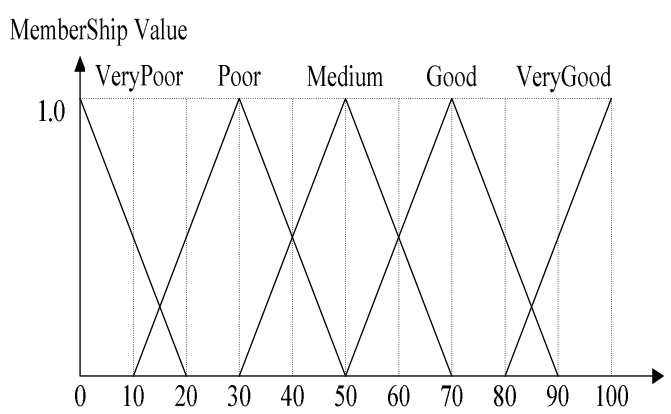

Figure 3. Membership function

In reality, multiple inter-dependences between criteria exist. Issues can complement or substitute each other. Complementary relationship between issues means that the two issues are needed together. In other words, the two issues increase together will lead to a more satisfactory bid for the decision maker. Substituted relationship between issues means that one issue can replace another. That is, even if only one issue increases, it can also result in a more satisfactory bid. In the partner selection process for automobile manufacturer, the price issue and the delivery issue complement each other, while the quality issue and the service issue substitute each other. Thus on one hand, a proposal which increase both price issue value and delivery issue value is more satisfactory for automobile manufacturer. On the other hand, a proposal which increases either quality issue value or service issue value is more satisfactory.

\section{B. Utility function}

To make tradeoffs across issues, the four issues are negotiated as a package deal. In each negotiation round, an agent submits a proposal which is a vector of the four issue values. The utility function is the representation of an agent's preferences by a mathematical function, which maps values of issues to the utility of bids. The notations used in the proposed utility function are listed as follows.

$m \in[1,4]$ denotes the number of negotiation issues,

$i \in[1, m]$ is the index set of negotiation issue,

$I_{i} \in\left[I_{i \min }, I_{i \max }\right]$ denotes the value of the $i^{t h}$ issue,

$b=<I_{1}, I_{2}, I_{3}, I_{4}>$ denotes the proposal (bid), 
$w_{i}$ denotes the relative importance of the $i^{t h}$ issue,

$u(I)$ denotes the normalization function of a issue,

$U(b)$ denotes the utility function of a proposal(bid).

In this paper, $\{1,2,3,4\}$ corresponds with \{quality, price, delivery, service\}. For instance, $I_{1}$ is the value of the quality issue and $w_{3}$ is the relative importance of the delivery issue. In order to compute conveniently, in this paper, we normalize all the four issues into 0-1 scale. The normalized value of issues can be obtained by commonly used linear transformation shown as (1).

$$
u\left(I_{i}\right)=\frac{I_{i}-I_{i \min }}{I_{i \max }-I_{i \min }}
$$

The utility of a partner is expressed as the weighted sum of normalized criteria. The result maybe depended on the weight of each criterion. In this paper, decision maker can specify the relative importance of criteria according to actual need. From automobile manufacturer's point of view, the four issues have a relative importance sequence of $w_{1} \succ w_{2} \succ w_{3} \succ w_{4}$. For ease of interpretation, we assume that the weights are nonnegative and are normalized, so $\sum_{i=1}^{m} w_{i}=1$.

Researchers usually assume that the multiple issues are independent and adopt a linear additive utility function to evaluate bids. In this paper, from automobile manufacturer's point of view, the price issue and the delivery issue have complementary relationship while the quality issue and the service issue have substituted relationship. In order to mimic these real world situations and generate more reasonable selection results, a non-linear utility function as shown in (2) is proposed to model the inter-dependences between multiple issues.

$$
\begin{aligned}
U(b)= & U\left(u\left(I_{2}\right), u\left(I_{3}\right)\right) \\
& +U\left(u\left(I_{1}\right), u\left(I_{4}\right)\right)
\end{aligned}
$$

The complementary relationship between the price issue and the delivery issue can be reflected by perfect complements utility function[10] shown as (3). $u\left(I_{2}\right)$ and $u\left(I_{3}\right)$ are the normalized value of the price issue and the delivery issue, $w_{2}$ and $w_{3}$ are the relative importance of the price issue and the delivery issue. As shown in Fig. 4, the utility can be increased only by increasing the normalized value of the price issue and the delivery issue together. This relationship corresponds with automobile manufacturer's attitude to the price issue and the delivery issue. So perfect complements utility function is adopted to reflect the inter-dependence between the price issue and the delivery issue.

$$
\begin{aligned}
& U\left(u\left(I_{2}\right), u\left(I_{3}\right)\right) \\
& =\min \left\{w_{2} u\left(I_{2}\right), w_{3} u\left(I_{3}\right)\right\}
\end{aligned}
$$

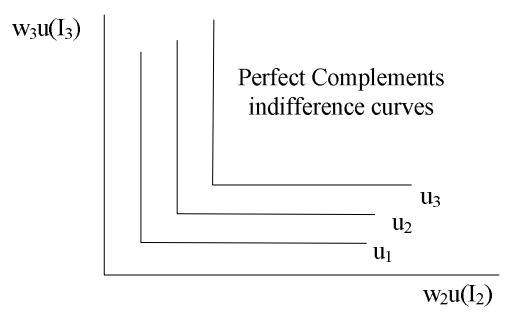

Figure 4. Perfect Complements utility function

The substituted relationship between the quality issue and the service issue can be reflected by perfect substitutes utility function[10] shown as (4). $u\left(I_{1}\right)$ and $u\left(I_{4}\right)$ denote the normalized value of the quality issue and the service issue, $w_{1}$ and $w_{4}$ indicate the relative importance of the quality issue and the service issue. As shown in Fig. 5, either the normalized value of the quality issue or the normalized value of the service issue increases can lead to the increase of utility. This relationship corresponds with automobile manufacturer's attitude to the quality issue and the service issue. So perfect substitutes utility function is adopted to reflect the interdependence between the quality issue and the service issue.

$$
\begin{aligned}
& U\left(u\left(I_{1}\right), u\left(I_{4}\right)\right) \\
& =w_{1} u\left(I_{1}\right)+w_{4} u\left(I_{4}\right)
\end{aligned}
$$

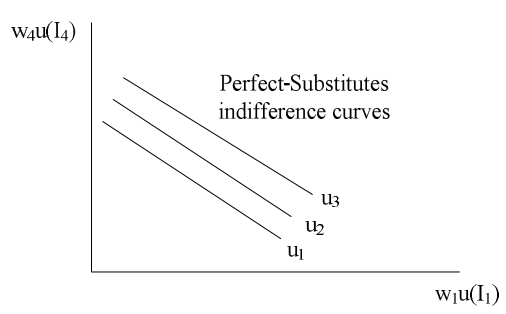

Figure 5. Perfect Substitutes utility function

The proposed non-linear utility function as shown in Fig. 6 can be obtained based on the hybrid of the perfect complements utility function and the perfect substitutes utility function. Equation (5) is the non-linear utility function on buyer's side.

$$
\begin{aligned}
U(b)= & \min \left\{w_{2} u\left(I_{2}\right), w_{3} u\left(I_{3}\right)\right\} \\
& +w_{1} u\left(I_{1}\right)+w_{4} u\left(I_{4}\right) \\
= & \min \left\{w_{2} u\left(I_{2}\right)+w_{1} u\left(I_{1}\right)+w_{4} u\left(I_{4}\right),\right. \\
& \left.w_{3} u\left(I_{3}\right)+w_{1} u\left(I_{1}\right)+w_{4} u\left(I_{4}\right)\right\}
\end{aligned}
$$




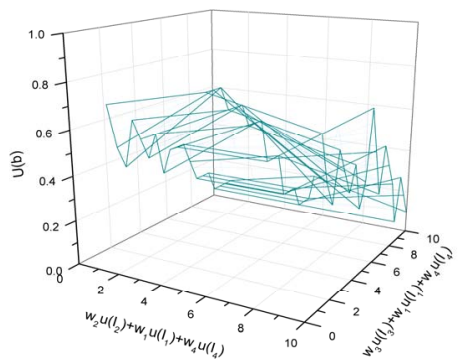

Figure 6. Example of proposed utility function

\section{Negotiation protocol and strategies}

Negotiation Protocols are the set of rules that govern the interaction of agents. It includes the participants, the negotiation states, the events that cause negotiation states to change and the valid actions of the participants in particular states. A nested protocol of FIPA-Contract-Net protocol and alternating-offer protocol is adopted in the proposed negotiation model. In the negotiation process agents act as proposer and responder to interact.

In this paper, we assume that both buyer agent and seller agent have time deadline. They must complete the negotiation before the deadline. In real outsourcing, buyer is more sensitive to time than sellers, so buyer agent adopts a time-dependent conceding strategy. In comparison, seller agents are not so sensitive to time, so they can adopt either time-dependent conceding strategy or fixed conceding strategy. The responding strategy directly depends on the conceding strategy. An agent can compare the utility of the current bid made by the opponent with its threshold derived by conceding strategies. If the utility of the current bid is higher, the agent accepts the bid; otherwise, the agent rejects it and propose and a counter-offer.

\section{CURRENT IMPLEMENTATION AND EXMPERIMENTS RESULT}

The proposed negotiation-based MAS framework has been implemented on JADE (Java Agent Development Framework) which is probably the most widespread agent-oriented middleware in use today. The basic functionalities of the proposed agent-based negotiation model are implemented. A database is created using MySQL to store seller's products information and negotiation data. The experiments conducted are to test the feasibility and performance of the proposed negotiation model supporting partner selection in a VE.

In this experiment, decision makers set their preferences by interface agents according to their needs. BA sends outsourcing auto parts information to $\mathrm{CA}$. Then $\mathrm{CA}$ selects potential partners for each auto part. And then BA and SA start one-toone negotiation for each auto part. Fig. 7 shows the interaction of agents in the proposed negotiation model that supporting auto parts partner selection for automobile manufacturer. In this negotiation process, automobile manufacturer selected one seller from three potential partners which are screened for this auto part by the optimization procedure. Table II shows the initial bids of the three potential partners. Table III, IV, and V show the negotiation results using the proposed agent-based negotiation model considering inter-dependences between issues when negotiation deadline is 40 rounds, 100 rounds and 200 rounds respectively.

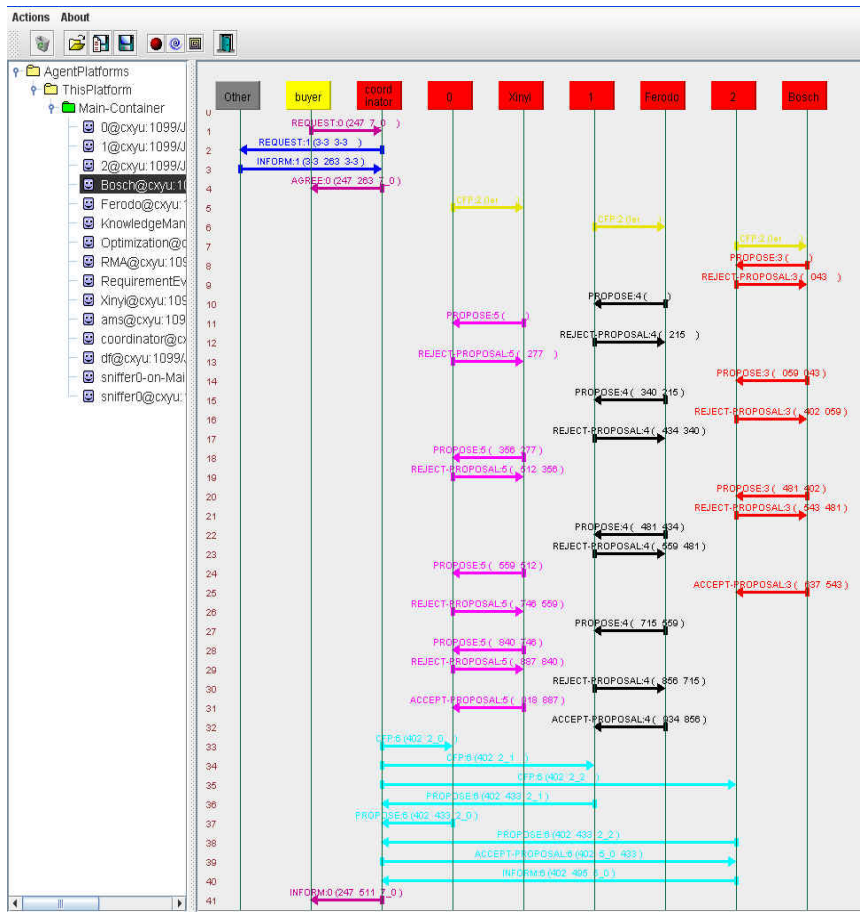

Figure 7. Agent negotiation tracing diagram

TABLE II. INITIAL BIDS OF EACH SELLER

\begin{tabular}{|c|c|}
\hline Seller & Bid \\
\hline S1 & $(950.0$, very good, 3, very good $)$ \\
\hline S2 & $(940.0$, very good, 5, very good $)$ \\
\hline S3 & $(810.0$, good, 10, very good $)$ \\
\hline
\end{tabular}

TABLE III. EXPERIMENT RESULTS $($ ROUND $=40)$

\begin{tabular}{|c|c|c|c|c|}
\hline Seller & $\begin{array}{c}\text { Time } \\
\text { (round) }\end{array}$ & Bid & $\begin{array}{c}\text { Buyer's } \\
\text { utility }\end{array}$ & $\begin{array}{c}\text { Seller's } \\
\text { utility }\end{array}$ \\
\hline S1 & 4 & $\begin{array}{c}\text { (575.0, very good, } \\
5, \text { very good) }\end{array}$ & 0.591 & 0.36 \\
\hline S2 & 4 & $\begin{array}{c}\text { (575.0, very good, } \\
5, \text { good) }\end{array}$ & 0.588 & 0.366 \\
\hline S3 & $\begin{array}{c}b \rightarrow s \\
s \rightarrow b\end{array}$ & $\begin{array}{c}\text { (749.25, good, } 8, \\
\text { very good) }\end{array}$ & 0.582 & 0.648 \\
\hline
\end{tabular}

TABLE IV. EXPERIMENT RESULTS $($ ROUND $=100)$

\begin{tabular}{|c|c|c|c|c|}
\hline Seller & $\begin{array}{c}\text { Time } \\
\text { (round) }\end{array}$ & Bid & $\begin{array}{c}\text { Buyer's } \\
\text { utility }\end{array}$ & $\begin{array}{c}\text { Seller's } \\
\text { utility }\end{array}$ \\
\hline S1 & $\begin{array}{c}8 \\
b \rightarrow s\end{array}$ & $\begin{array}{c}(570.0, \text { very good, } \\
5, \text { very good) }\end{array}$ & 0.592 & 0.356 \\
\hline S2 & 8 & $\begin{array}{c}(570.0, \text { very good, } \\
5, \text { good) }\end{array}$ & 0.590 & 0.361 \\
\hline S3 & $\begin{array}{c}13 \\
s \rightarrow b\end{array}$ & $\begin{array}{c}\text { (761.4, good, } 8, \\
\text { very good) }\end{array}$ & 0.579 & 0.660 \\
\hline
\end{tabular}


TABLE V. EXPERIMENT RESULTS $($ ROUND $=200)$

\begin{tabular}{|c|c|c|c|c|}
\hline Seller & $\begin{array}{c}\text { Time } \\
\text { (round) }\end{array}$ & Bid & $\begin{array}{c}\text { Buyer's } \\
\text { utility }\end{array}$ & $\begin{array}{c}\text { Seller's } \\
\text { utility }\end{array}$ \\
\hline S1 & $\begin{array}{c}12 \\
b \rightarrow s\end{array}$ & $\begin{array}{c}(555.0, \text { very good, } \\
\text { 4, very good) }\end{array}$ & 0.595 & 0.337 \\
\hline S2 & 16 & $\begin{array}{c}(575.0, \text { very good, } \\
5, \text { good) }\end{array}$ & 0.588 & 0.366 \\
\hline S3 & $\begin{array}{c}25 \\
s \rightarrow b\end{array}$ & $\begin{array}{c}\text { (761.4, good, } 8, \\
\text { very good) }\end{array}$ & 0.579 & 0.660 \\
& $S \quad$ & & \\
\hline
\end{tabular}

According to the negotiation results in Table III, IV, and V, the proposed negotiation model is feasible and S1 is the best partner in this VE. In addition, the comparison of negotiation results under different deadline shown as Table III, IV, and V indicates that longer negotiation time can reach better negotiation result (550.0, very good, 4, very good). That is, the proposed negotiation model can obtain better performance on longer time.

\section{CONCLUSION AND FUTURE WORK}

This paper presents an agent-based negotiation model to support partner selection in a VE. In this model, both VE initiator and potential partners can discuss their proposals by negotiation. In the negotiation process, not only multiple issues are considered, but also the inter-dependences between issues are considered. The multiple issues can be expressed both in qualitative and quantitative forms. The negotiation process is controlled by a nested protocol of FIPA-Contract-Net protocol and alternating-offer protocol. VE initiator and potential partners can use time-dependent or fixed conceding strategy according to their situation.

The agent-based negotiation model supporting partner selection in a VE is implemented and the feasibility of the proposed model is tested. For future work, more experiments will be conducted to test the efficiency and effectiveness of the proposed model supporting partner selection for several auto parts simultaneously. In addition, the determination of relative importance of issues considering inter-dependences between issues will be further studied. Finally the method that supports agents to choose appropriate negotiation strategies automatically will be further studied.

\section{REFERENCES}

[1] Camarinha-Matos, L.M. and H. Afsarmanesh, Elements of a base VE infrastructure. Computers in Industry, 2003. 51: p. 139-163.

[2] Dickson, G.W., An analysis of vendor selection systems and decisions. Journal of Purchasing, 1966. 2(1): p. 5-17.

[3] Weber, C.A., J.R. Current, and W.C. Benton, Vendor selection criteria and methods. European Journal of Operational Research, 1991. 50: p. 218.

[4] Zhang, Z., et al., Evolution of Supplier Selection Criteria and Methods. European Journal of Operational Research, 2003.

[5] Ip, W., et al., Genetic algorithm solution for a risk-based partner selection problem in a virtual enterprise. Computers and Operations Research, 2003. 30(2): p. 213-231.

[6] Sha, D. and Z. Che, Virtual integration with a multi-criteria partner selection model for the multi-echelon manufacturing system. The International Journal of Advanced Manufacturing Technology, 2005. 25(7): p. 793-802.

[7] Sari, B., T. Sen, and S. Kilic, Ahp model for the selection of partner companies in virtual enterprises. The International Journal of Advanced Manufacturing Technology, 2008. 38(3): p. 367-376.

[8] Jarimo, T. and A. Salo, Multi-Criteria Partner Selection in Virtual Organisations With Transportation Costs and Other Network Interdependencies. IEEE Transactions on Systems, Man, and Cybernetics, Part C: Applications and Reviews, 2009. 39(1): p. 124-129.

[9] Camarinha-Matos, L.M. and H. Afsarmanesh, Virtual enterprise modeling and support infrastructures: applying multi-agent system approaches, in Multi-Agent Systems and Applications. 2001, Springer: London, UK. p. 335-364.

[10] Besanko, D.A. and R.R. Braeutigam, Microeconomics : an integrated approach 2002, New York 81-107. 